\title{
Vapor-liquid equilibria and conceptual design of extractive distillation for separating ethanol and ethyl propionate
}

Yunfei Song, Yuezhan Du, Ruyue Wang, Hongze Yan, Fei Luo, Lanyi Sun*

State Key Laboratory of Heavy Oil Processing, College of Chemical Engineering, China

University of Petroleum (East China), Qingdao Shandong 266580, China 
Table S1. Parameters of Antoine equation

\begin{tabular}{cccccccccc}
\hline & $\mathrm{C}_{1 \mathrm{i}}$ & $\mathrm{C}_{12 \mathrm{i}}$ & $\mathrm{C}_{3 \mathrm{i}}$ & $\mathrm{C}_{4 \mathrm{i}}$ & $\mathrm{C}_{5 \mathrm{i}}$ & $\mathrm{C}_{6 \mathrm{i}}$ & $\mathrm{C}_{7 \mathrm{i}}$ & $\mathrm{C}_{8 \mathrm{i}}$ & $\mathrm{C}_{9 \mathrm{i}}$ \\
\hline ethanol & 66.3962 & -7122.3 & 0 & 0 & -7.1424 & $2.89 \mathrm{E}-6$ & 2 & 159.05 & 514 \\
$\begin{array}{c}\text { Ethyl } \\
\text { propionate } \\
\begin{array}{c}\text { Isobutyl } \\
\text { acetate }\end{array}\end{array}$ & 65.4022 & -6944.3 & 0 & 0 & -7.298 & $3.79 \mathrm{E}-6$ & 2 & 174.3 & 561 \\
\hline
\end{tabular}


Table S2. van der Waals areas $(q)$ and volumes $(r)$ of pure components for the UNIQUAC model

\begin{tabular}{cccc}
\hline & Ethanol & Ethyl propionate & Isobutyl acetate \\
\hline$q$ & 1.972 & 3.656 & 4.192 \\
$r$ & 2.10547 & 4.15293 & 4.82663 \\
\hline
\end{tabular}


Table S3. Liquid molar volume of pure components for the Wilson equation.

\begin{tabular}{llll}
\hline & Ethanol & Ethyl propionate & Isobutyl acetate \\
\hline$V_{L}(\mathrm{ml} / \mathrm{mol})$ & 58.6197 & 133.811 & 115.622 \\
\hline
\end{tabular}


Table S4. The binary parameters for ethanol + ethyl propionate

\begin{tabular}{lllll}
\hline \multirow{2}{*}{ Model } & \multicolumn{4}{c}{ Binary parameters } \\
\cline { 2 - 5 } & $\boldsymbol{a}_{\boldsymbol{i j}}$ & $\boldsymbol{a}_{\boldsymbol{j} \boldsymbol{i}}$ & $\boldsymbol{b}_{\boldsymbol{i j}}$ & $\boldsymbol{b}_{\boldsymbol{j} \boldsymbol{i}}$ \\
\hline NRTL & 0 & 0 & 398.01 & -16.592 \\
UNIQUAC & 0 & 0 & 12.475 & -180.58 \\
Wilson & 0 & 0 & -53.459 & -342.106 \\
\hline
\end{tabular}


Table S5. Cost estimating formulas for TAC.

\begin{tabular}{|c|c|}
\hline Project & Formula \\
\hline Column diameter (D) & Aspen tray sizing \\
\hline Column length $(\mathrm{H})$ & $(\mathrm{NT}-2) \times 0.61 \times 1.2$ \\
\hline column shell cost $(\$)$ & $\begin{array}{l}(\mathrm{CEPCI} / 119)\left(957.9 \mathrm{D}^{1.066} \mathrm{H}^{0.802}\right)\left(2.18+\mathrm{F}_{\mathrm{m}} \mathrm{F}_{\mathrm{p}}\right) \text {, where } \\
\text { the coefficient } \mathrm{F}_{\mathrm{m}}=1, \mathrm{~F}_{\mathrm{p}}=0.983 \text {. }\end{array}$ \\
\hline column tray cost $(\$)$ & $\begin{array}{l}(\mathrm{CEPCI} / 119)\left(97.2 \mathrm{D}^{1.55} \mathrm{H}\right)\left(\mathrm{F}_{\mathrm{t}}+\mathrm{F}_{\mathrm{m}}\right) \text {, where the } \\
\text { coefficient } \mathrm{F}_{\mathrm{t}}=0 \text {. }\end{array}$ \\
\hline heat exchange area $\left(\mathrm{m}^{2}\right)$ & $\begin{array}{l}\mathrm{A}=\mathrm{Q} /\left(\mathrm{U}^{*} \Delta \mathrm{T}\right) \text {, where } \mathrm{Q} \text { is the duty of the heat } \\
\text { exchanger; } \mathrm{U} \text { is the heat transfer coefficient; } \Delta \mathrm{T} \text { is the } \\
\text { temperature difference. }\end{array}$ \\
\hline Heat-transfer coefficient (Condensers) & $\mathrm{U}=0.852 \mathrm{~kW} / \mathrm{K}-\mathrm{m}^{2}$ \\
\hline Heat-transfer coefficient (Reboilers) & $\mathrm{U}=0.568 \mathrm{~kW} / \mathrm{K}-\mathrm{m}^{2}$ \\
\hline \multirow[t]{3}{*}{ heat exchanger cost $(\$)$} & $(\mathrm{CEPCI} / 119)\left(474.7 \mathrm{~A}^{0.65}\right)\left[2.29+\mathrm{F}_{\mathrm{m}}\left(\mathrm{F}_{\mathrm{p}}+\mathrm{F}_{\mathrm{t}}\right)\right]$, where \\
\hline & the coefficient $\mathrm{Fp}=0 ; \mathrm{Fd}=1.35$ for kettle reboiler and \\
\hline & 0.8 for fixed-tube sheet \\
\hline LP steam $\left(5\right.$ barg, $\left.160{ }^{\circ} \mathrm{C}\right)$ & $\$ 7.78 / \mathrm{GJ}$ \\
\hline Cooling water $(320 \mathrm{~K})$ & $\$ 0.354 / \mathrm{GJ}$ \\
\hline
\end{tabular}




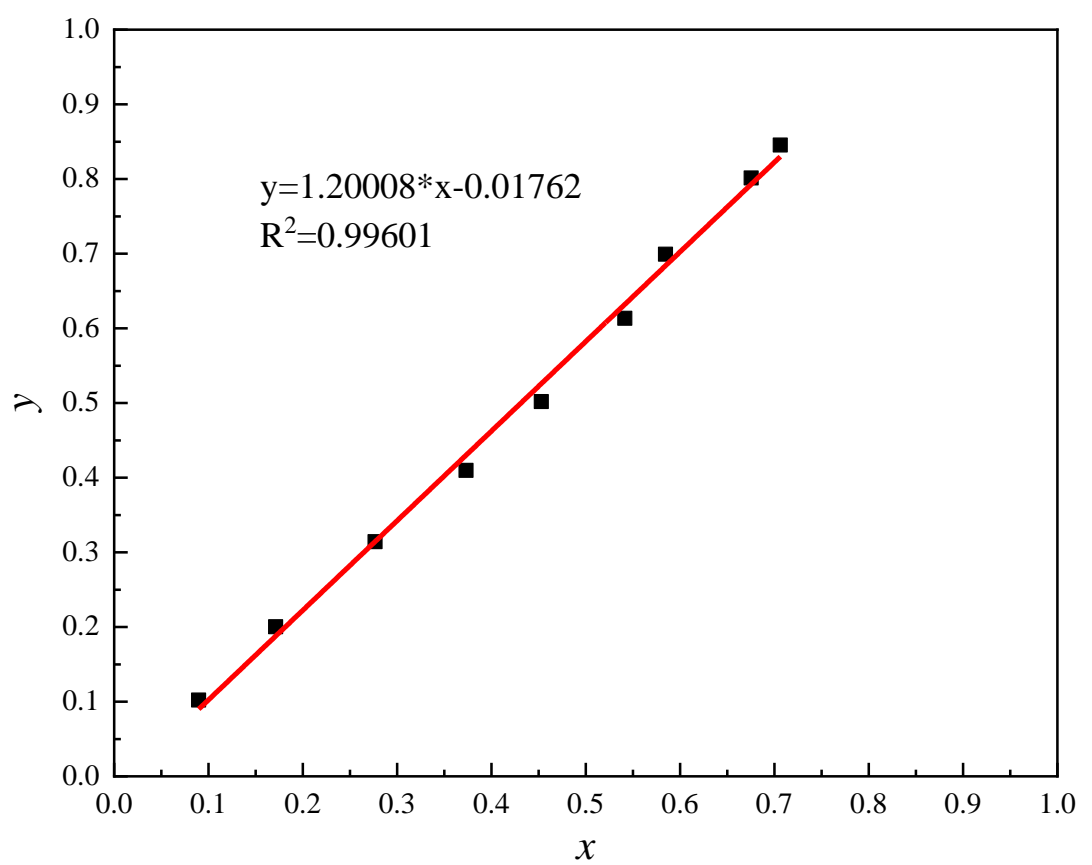

(a)

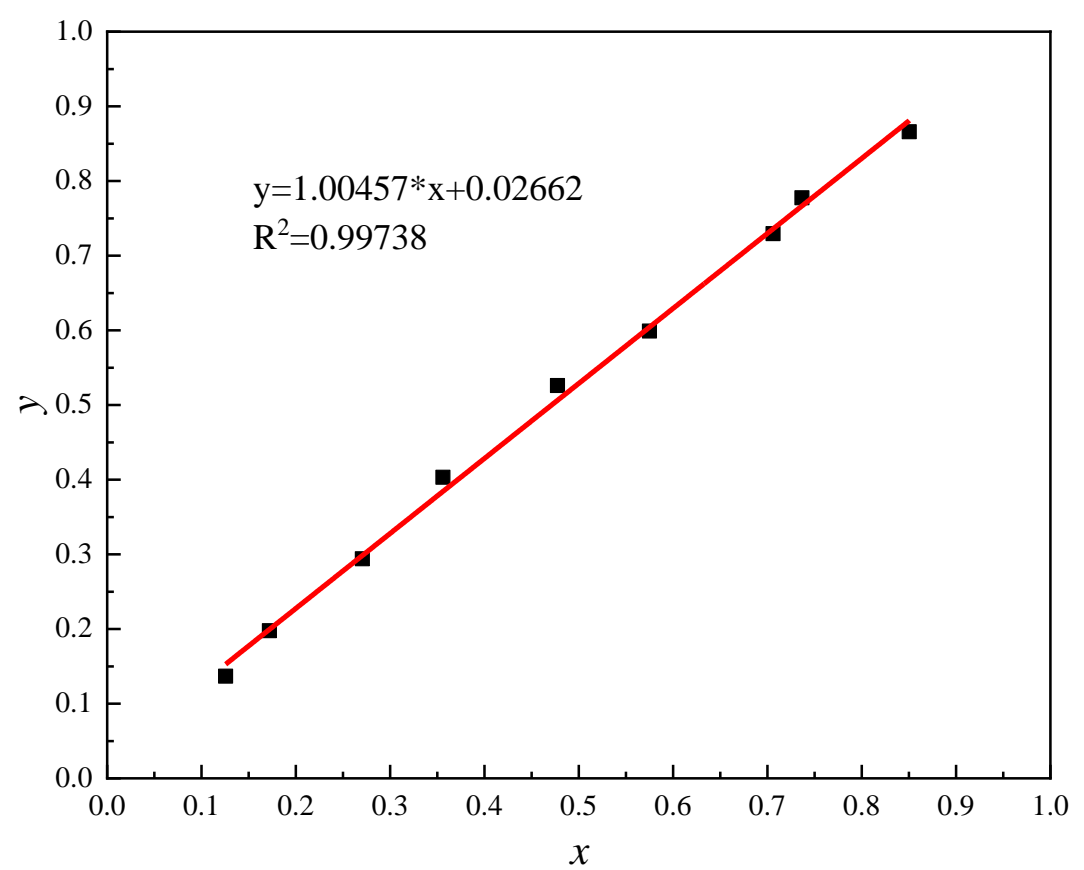

(b)

Figure S1. Calibration curves for (a) ethanol + isobutyl acetate; (b) ethyl propionate + isobutyl acetate binary systems. 


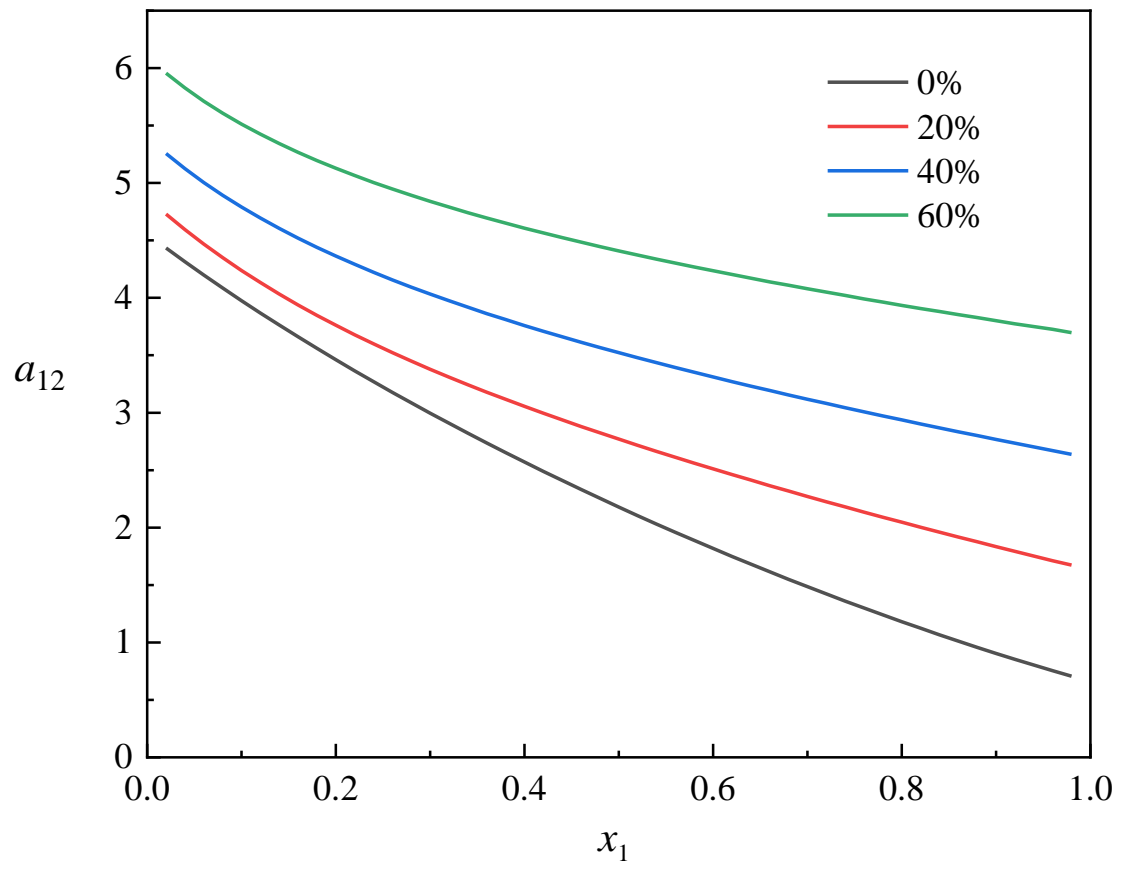

Figure S2. Relative volatility for ethanol (1) + ethyl propionate (2) binary system at $101.3 \mathrm{kPa}$ 